\title{
What makes you can also break you: mitochondrial permeability transition pore formation by the c subunit of the $\mathrm{F}_{1} \mathrm{~F}_{0}$ ATP-synthase?
}

\author{
Christos Chinopoulos ${ }^{1}$ and Gyorgy Szabadkai ${ }^{2,3 *}$ \\ 1 Department of Medical Biochemistry, Semmelweis University, Budapest, Hungary \\ ${ }^{2}$ Department of Cell and Developmental Biology, Consortium for Mitochondrial Research, University College London, London, UK \\ ${ }^{3}$ Department of Biomedical Sciences, University of Padua, Padua, Italy \\ *Correspondence: g.szabadkai@ucl.ac.uk
}

Edited by:

Lorenzo Galluzzi, Institut National de la Santé et de la Recherche Medicale, France

Reviewed by:

Lorenzo Galluzzi, Institut National de la Santé et de la Recherche Medicale, France

\section{A commentary on}

Role of the $c$ subunit of the F0 ATP synthase in mitochondrial permeability transition

by Bonora, M., Bononi, A., De Marchi, E., Giorgi, C., Lebiedzinska, M., Marchi, S., et al. (2013). Cell Cycle 12, 674-683.

A number of cellular stresses and cytotoxic agents trigger mitochondrial permeability transition (mPT), considered as a final common pathway of cell death (Brenner and Grimm, 2006). mPT follows the formation of a large non-selective pore (mPTP) in the inner membrane of mitochondria (IMM), permeable to molecules up to $1.5 \mathrm{kD}$. Accordingly, mPT leads to metabolic insufficiency of mitochondria (Chinopoulos and Adam-Vizi, 2010) and has been assumed to underlie the induction of both accidental (necrotic) and regulated forms of cell death (e.g. the intrinsic apoptotic or necroptotic pathways) (Galluzzi et al., 2012). Indeed, pharmacological and posttranslational modification of cyclophilin-D (cypD), an established regulatory subunit of the mPTP, has been shown to modulate cell sensitivity to death induction in several pathologies (Giorgio et al., 2011). Thus, the pore will likely represent the target of a novel regulated cell death modality but the lack of information on its molecular identity is persistently impeding the characterization of this pathologically highly relevant pathway.

Recently, cypD has been shown to interact with and regulate the $\mathrm{F}_{1} \mathrm{~F}_{0}$ ATP-synthase, the main molecular motor of chemiosmotic ATP production in the mitochondrion (Giorgio et al., 2009; Chinopoulos et al., 2011), raising the odd suspicion that the most fundamental pillar of cellular energy metabolism might also be the gatekeeper, or even the principal component of mPT (Chinopoulos and Adam-Vizi, 2012). The first experimental trial of this curious idea has now emerged in the latest issue of Cell Cycle (Bonora et al., 2013). The study addressed the effect of the overexpression and silencing of the ring-forming subunit $\mathrm{c}$ of the membrane spanning $\mathrm{F}_{0}$ unit, and demonstrated that the propensity of $\mathrm{mPT}$ highly correlates with the subunit c expression levels. These results imply that a conformational change of the c-ring might transform it to a nonselective pore, presenting a provocative idea leading to a series of outstanding questions.

The first, conceptually most challenging problem is how to separate the ATP-synthase and eventual pore-forming activity of the $\mathrm{F}_{1} \mathrm{~F}_{0}$ complex in the experimental design. The rotation of the membrane-embedded ring formed by the $c$ subunits of the ATP-synthase is driven by the proton motive force across the IMM. Since, according to the current model, the protonation/de-protonation cycle of each $c$ subunit is required for the translocation of one $\mathrm{H}^{+}$, and a complete $360^{\circ}$ rotation of the ring generates 3 ATP molecules on the $\alpha$ catalytic subunit, the number of $\mathrm{c}$ subunits will dictate the bioenergetic cost of making one ATP per $\mathrm{F}_{1} \mathrm{~F}_{0}$ ATP-synthase (Watt et al., 2010). Knockdown of the $c$ subunit can either decrease the number of $c$ subunits per
$\mathrm{F}_{1} \mathrm{~F}_{0}$ ATP-synthase molecule, or reduce the overall number of functional $\mathrm{F}_{1} \mathrm{~F}_{0}$ ATP-synthases, but in both cases it would alter the efficiency of the ATP hydrolysis or production at a given mitochondrial membrane potential. Thus, separating the consequent bioenergetic effects from the direct molecular consequences of $c$ subunit knockdown is essentially unworkable. The authors addressed this issue by silencing the catalytic F1-localized $\alpha$ subunit as a control, which had no effect on mPT, while the genetic manipulation should have had the same impact on the number of functional $\mathrm{F}_{1} \mathrm{~F}_{0}$ ATP-synthases. This indicates that the observed effect on $\mathrm{mPT}$ is indeed specific to the c subunit, but the detailed characterization of an eventually altered stoichiometry of the $\mathrm{F}_{1} \mathrm{~F}_{0}$ complex on the $\mathrm{ATP} / \mathrm{H}^{+}$(or the $\mathrm{P} / \mathrm{O}$ ) ratio warrants further analysis, particularly in view of the fact that the number of copies of subunit $c$ is constant and appears to be independent of the metabolic state within all vertebrate animals and most invertebrate species (Watt et al., 2010).

The second, technically challenging question is whether the $\mathrm{F}_{0} \mathrm{c}$-ring can form a pore with characteristics of the $\mathrm{MPTP}$ ? Would other subunits also be required? The idea might not be too far-fetched, since it has been shown that subunit $c$, reconstituted in lipid bilayers, forms a voltage sensitive pore mediating a $\mathrm{Ca}^{2+}$. regulated cation current (McGeoch et al., 2000). Moreover, elastic network modeling inferred that the c-ring exhibits significant flexibility allowing for some extreme deformations during operation of the 
$\mathrm{F}_{1} \mathrm{~F}_{0}$ ATP-synthase (Saroussi et al., 2012). Again, whether an mPTP-like pore can be formed this way, and if yes, under what conditions/composition will need further rigorous testing.

Finally, these findings recall an old question about the pharmacology of mPT. Oligomycin, a potent inhibitor of the $\mathrm{F}_{1} \mathrm{~F}_{0}$ ATP-synthase, targets the $c$ subunit, suggesting that it might also affect mPTP formation. Whilst many early studies on isolated mitochondria have shown $\mathrm{mPT}$ inhibition by oligomycin, the effect was always accounted for changes in ATP/ADP concentrations, locking the pore in a closed conformation. We are aware only one study so far addressing the direct effect of oligomycin on mPT-induced cell death. Shchepina et al. have shown that whilst oligomycin (acting on the $\mathrm{F}_{0} \mathrm{c}$ subunits) was able to almost completely block TNF/emetine-induced cell death, aurovertin B (inhibiting ATP synthesis on the $F_{1}$ catalytic subunits) had no effect on $\mathrm{mPT}$ in this cell death modality (Shchepina et al., 2002). This result echoes the findings of Bonora et al. (2013) but yet again provokes further questions. First, can the mPTP forming abilities of the $c$ subunits be specifically targeted without compromising cell viability? Second, while oligomycin efficiently blocked TNF/emetin-induced cell death, it was ineffective against staurosporineinduced apoptosis, suggesting the existence of a specific oligomycin-sensitive (c subunit-mediated) cell death modality. Will this modality fit into the catalogue of the known biochemically characterized cell death pathways (e.g., necroptosis) or will it represent an entirely novel death subroutine?
Altogether, as shown by the above list of outstanding questions, we anticipate that the findings by Bonora et al. (2013) are only the beginning of a new shakeup in the mitochondrial field, and more detailed analyses of the role of the $\mathrm{F}_{1} \mathrm{~F}_{0}$ ATP-synthase in $\mathrm{MPT}$ and cell death will soon follow.

\section{ACKNOWLEDGMENTS}

Christos Chinopoulos is supported by the Hungarian Academy of Sciences (MTA-SE Lendület Neurobiochemistry Research Division grant 95003) and the Hungarian Scientific Research Fund (grant K 100918). Gyorgy Szabadkai is supported by Parkinson's UK, Wellcome Trust, Italian Association for Cancer Research (AIRC) and Telethon Italy.

\section{REFERENCES}

Bonora, M., Bononi, A., De Marchi, E., Giorgi, C., Lebiedzinska, M., Marchi, S., et al. (2013). Role of the $c$ subunit of the F0 ATP synthase in mitochondrial permeability transition. Cell Cycle 12, 674-683.

Brenner, C., and Grimm, S. (2006). The permeability transition pore complex in cancer cell death. Oncogene 25, 4744-4756.

Chinopoulos, C., and Adam-Vizi, V. (2010). Mitochondria as ATP consumers in cellular pathology. Biochim. Biophys. Acta 1802, 221-227.

Chinopoulos, C., and Adam-Vizi, V. (2012). Modulation of the mitochondrial permeability transition by cyclophilin $\mathrm{D}$ : moving closer to $\mathrm{F}(0)-\mathrm{F}(1)$ ATP synthase? Mitochondrion 12, 41-45.

Chinopoulos, C., Konràd, C., Kiss, G., Metelkin, E., Töröcsik, B., Zhang, S. F., et al. (2011). Modulation of F0F1-ATP synthase activity by cyclophilin D regulates matrix adenine nucleotide levels. FEBS J. 278, 1112-1125.

Galluzzi, L., Vitale, I., Abrams, J. M., Alnemri, E. S., Baehrecke, E. H., Blagosklonny, M. V., et al. (2012). Molecular definitions of cell death subroutines: recommendations of the Nomenclature
Committee on Cell Death 2012. Cell Death Differ. 19, 107-120.

Giorgio, V., Bisetto, E., Soriano, M. E., DabbeniSala, F., Basso, E., Petronilli, V., et al. (2009). Cyclophilin D modulates mitochondrial F0F1ATP synthase by interacting with the lateral stalk of the complex. J. Biol. Chem. 284, 33982-33988.

Giorgio, V., Soriano, M. E., Basso, E., Bisetto, E., Lippe, G., Forte, M. A., et al. (2011). Cyclophilin $\mathrm{D}$ in mitochondrial pathophysiology. Biochim. Biophys. Acta 1797, 1113-1118.

McGeoch, J. E., McGeoch, M. W., Mao, R., and Guidotti, G. (2000). Opposing actions of cGMP and calcium on the conductance of the $\mathrm{F}(0)$ subunit c pore. Biochem. Biophys. Res. Commun. 274, 835-840.

Saroussi, S., Schushan, M., Ben-Tal, N., Junge, W. and Nelson, N. (2012). Structure and flexibility of the C-ring in the electromotor of rotary F(0)F(1)-ATPase of pea chloroplasts. PLoS ONE 7:e43045. doi: 10.1371/journal.pone.0043045

Shchepina, L. A., Pletjushkina, O. Y., Avetisyan, A. V., Bakeeva, L. E., Fetisova, E. K., Izyumov, D. S., et al. (2002). Oligomycin, inhibitor of the F0 part of H+-ATP-synthase, suppresses the TNF-induced apoptosis. Oncogene 21, 8149-8157.

Watt, I. N., Montgomery, M. G., Runswick, M. J., Leslie, A. G. W., and Walker, J. E. (2010). Bioenergetic cost of making an adenosine triphosphate molecule in animal mitochondria. Proc. Natl. Acad. Sci. U.S.A. 107, 16823-16827.

Received: 01 February 2013; accepted: 01 February 2013; published online: 19 February 2013.

Citation: Chinopoulos C and Szabadkai G (2013) What makes you can also break you: mitochondrial permeability transition pore formation by the $c$ subunit of the $F_{1} F_{0}$ ATP-synthase? Front. Oncol. 3:25. doi: 10.3389/ fonc.2013.00025

This article was submitted to Frontiers in Molecular and Cellular Oncology, a specialty of Frontiers in Oncology. Copyright (c) 2013 Chinopoulos and Szabadkai. This is an open-access article distributed under the terms of the Creative Commons Attribution License, which permits use, distribution and reproduction in other forums, provided the original authors and source are credited and subject to any copyright notices concerning any third-party graphics etc. 